\title{
P20-06. Study of viral variability evolution in patients submitted to a therapeutic vaccine based on autologous dendritic cells pulsed with autologous HIV-I
}

\author{
M Guilà*1, J Dalmau ${ }^{2}$, C Gil1 ${ }^{1}$, J Martinez-Picado ${ }^{2}$, F García ${ }^{1}$, N Climent ${ }^{1}$, \\ M García ${ }^{1}$, C Hurtado${ }^{1}$, T Pumarola ${ }^{1}$, J Miró ${ }^{1}$, T Gallart ${ }^{1}$ and J Gatell ${ }^{1}$
}

\author{
Address: ${ }^{1}$ Retrovirology and Viral Immunopathogeny Laboratory, Hospital Clínic/IDIBAPS-HIVACAT, University of Barcelona, Barcelona, Spain \\ and 2Irsicaixa Foundation-HIVACAT, Hospital Germans Trias i Pujol, Badalona, Spain \\ * Corresponding author
}

from AIDS Vaccine 2009

Paris, France. 19-22 October 2009

Published: 22 October 2009

Retrovirology 2009, 6(Suppl 3):P376 doi:10.1 186/1742-4690-6-S3-P376

This abstract is available from: http://www.retrovirology.com/content/6/S3/P376

(c) 2009 Guilà et al; licensee BioMed Central Ltd.

\section{Background}

A previous therapeutic vaccine pilot study based on autologous dendritic cells pulsed with autologous heat inactivated viruses HIV-1 obtained by plasmapheresis, showed a decrease in the plasma viral load in 4 of 12 chronically HIV-1-infected patients [García et al., J Infect Dis. 2005; 191: 1680-1685]. This vaccination strategy required to submit the infected patients to 2 structured treatment interruptions (STI): the first to recover enough viruses to be used as antigen, and the second to assess virological responses after the immunization. We aimed to study the viral variability evolution changes occurring during off therapy period, before and after vaccination.

\section{Methods}

12 patients on HAART underwent a HAART interruption (STOP1) to obtain the virus by plasmaphereses. Then, HAART was reinitiated and thereafter they received 4 immunizations. Six weeks after the last immunization, HAART was definitely interrupted (STOP2). 4 controls were submitted to both HAART interruptions but did not receive any vaccination.

Sequences corresponding to the envelope C2V5 region were analyzed from plasma samples obtained at baseline, STOP1 and STOP2. Frequency and complexity values were manually calculated. Genetic distances were con- ducted using MEGA software version 4 [Tamura et al., Mol Biol Evol., 2007; 24: 1596-1599].

\section{Results}

A high complexity was observed in all patients. The divergence rate between stops was higher than between baseline and STOP1, and divergence rate between baseline and STOP 2 was lower than between baseline and STOP1.

In the immunized patients, the viral complexity was lower in STOP2 than in STOP1, and there was a trend to decrease amino acid changes and diversity after this therapeutic vaccination.

\section{Conclusion}

All patients showed a great variability in all studied populations. We suggest that viruses observed in STOP2 are more related to the baseline than with the viruses found in STOP1 in all patients. Immunized patients showed lower viral complexity after the therapeutic vaccination. 